\title{
Efficient CAD of Boxed Microwave Circuits Based on Arbitrary Rectangular Elements
}

\author{
Alejandro Alvarez Melcón, Juan R. Mosig, Fellow, IEEE, and Marco Guglielmi
}

\begin{abstract}
In this paper, we describe a very accurate and computationally efficient computer-aided design (CAD) tool for the analysis and design of a wide class of boxed microwave circuits composed of arbitrary rectangular elements printed on dielectric layers. The theoretical derivations are based on an integral equation formulation, and call for the evaluation of the boxed multilayer Green's functions, thus leading to a tool that is valid for an arbitrary number of circuits and dielectric layers. In addition to theory, comparisons with measured results are presented, and several practical filter structures are also investigated, thus clearly demonstrating that the CAD tool developed can indeed be used very effectively for the design of a large variety of microwave circuits.
\end{abstract}

Index Terms-Broadside coupled, integral equation, microwave filters, modal expansions, shielded printed circuits, transmission zeros.

\section{INTRODUCTION}

$\mathbf{T}$ HE current industrial design of modern microwave circuits requires strongly reduced development times and costs. As a result, the development of fast and efficient software tools that can accurately predict the electrical behavior of the components being investigated is of fundamental importance.

In this context, the modeling of boxed printed circuits and antennas has always attracted much attention (see, e.g., [1] and [2]). Of particular interest are the results in [3], where the effects of the lateral walls and of the top cover were studied. The results obtained in [3] clearly indicate that, for practical boxed circuits, the shielding effect can no longer be neglected. The presence of a metallic enclosure, however, introduces a discrete spectrum, and, as a consequence, the relevant physical quantities are expressed in terms of infinite series that are very slowly convergent.

To solve this problem, various accelerations techniques have been reported (see, e.g., [4]-[6]). Among the various techniques developed, of particular relevance is the work by Eleftheriades et al. [7], where the asymptotic part of the sums was extracted and transformed into a form involving the fast decaying modified Bessel function. In spite of all this effort, however, computational time still remains the limiting factor in the practical design of microwave circuits.

Manuscript received MAY 8, 1998.

A. Alvarez Melcón and J. R. Mosig are with the Electromagnetics and Acoustics Laboratory, Swiss Federal Institute of Technology, Ecole Polytechnique Fédérale de Lausanne, CH-1015 Lausanne, Switzerland.

M. Guglielmi is with the European Space Research and Technology Center, 2200AG Noordwijk, The Netherlands.

Publisher Item Identifier S 0018-9480(99)05309-0.
To reduce the computational effort required for the analysis of arbitrary boxed circuits, we propose in this paper to simplify the basic geometry, so that closed-form analytical formulations can be used, yielding far more accurate and efficient algorithms. The basic structure discussed in this paper is the one shown in Fig. 1, and is composed of an arbitrary number of rectangular elements of zero thickness printed on an arbitrary number of dielectric layers. The use of this basic geometry allows for the definition of very efficient current expansion functions on the metallic areas, which considerably accelerate the convergence rate of the series involved and lead to very efficient computer codes. It is important to note, however, that, in spite of the restriction imposed on the geometry, a very wide class of microwave circuits can still be analyzed.

The paper is organized as follows. In Section II, the basic formulation is presented. Section IV is devoted to the validation of the theory through comparison between simulated and measured results. Finally, in Section V, we describe how the tool developed can indeed be used to study a large variety of microwave structures of practical interest.

\section{INTEGRAL-EQUATION FORMULATION}

The general multilayer boxed printed circuit under examination is shown in Fig. 1. The analysis is performed via an integral-equation formulation. The formulation starts with the imposition of the boundary conditions for the fields in the structure. If we take $n$ arbitrary rectangular elements, with induced electric currents $\bar{J}_{s_{1}}, \bar{J}_{s_{2}}, \ldots, \bar{J}_{s_{n}}$ (Fig. 2), the suitable boundary condition is associated to the tangent electric field on the surfaces of the $n$ metallic areas

$$
\left.\bar{E}_{t_{\text {(tot) }}}\right|_{s_{e_{q}}}=Z_{s} \bar{J}_{s_{q}}, \quad 1 \leq q \leq n
$$

where the surface impedance $Z_{s}$ has been used to take into account the finite conductivity of the metal and, for good conductors, we have

$$
Z_{s}=(1+j) \sqrt{\frac{\omega \mu_{0}}{2 \sigma}} .
$$

Furthermore, in a practical circuit, there are always a number of ports used to introduce an excitation and one or more ports to extract power. As a consequence, an exciting (or impressed) field $\bar{E}_{t_{(e)}}$ needs to be defined on the lines acting as input ports. Once the circuit is excited, the scattered field $\bar{E}_{t_{\text {(scat) }}}$ will induce electric currents on all metallic areas. If we define a subset $(v)$ of the lines acting as ports, as shown in Fig. 2, the boundary conditions in (1) splits into two categories, one 


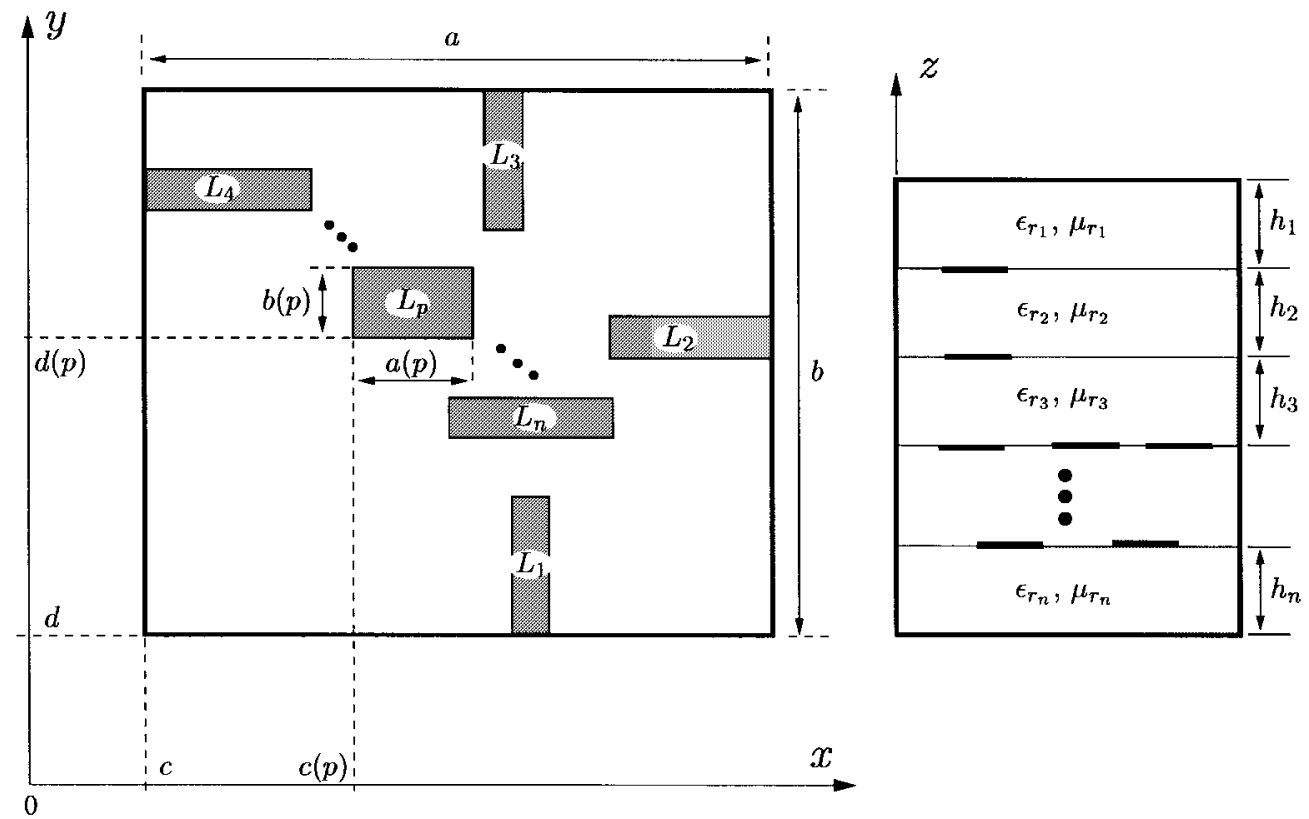

Fig. 1. General boxed multilayered microwave printed circuit composed of an arbitrary number of metallic lines analyzed in this paper.

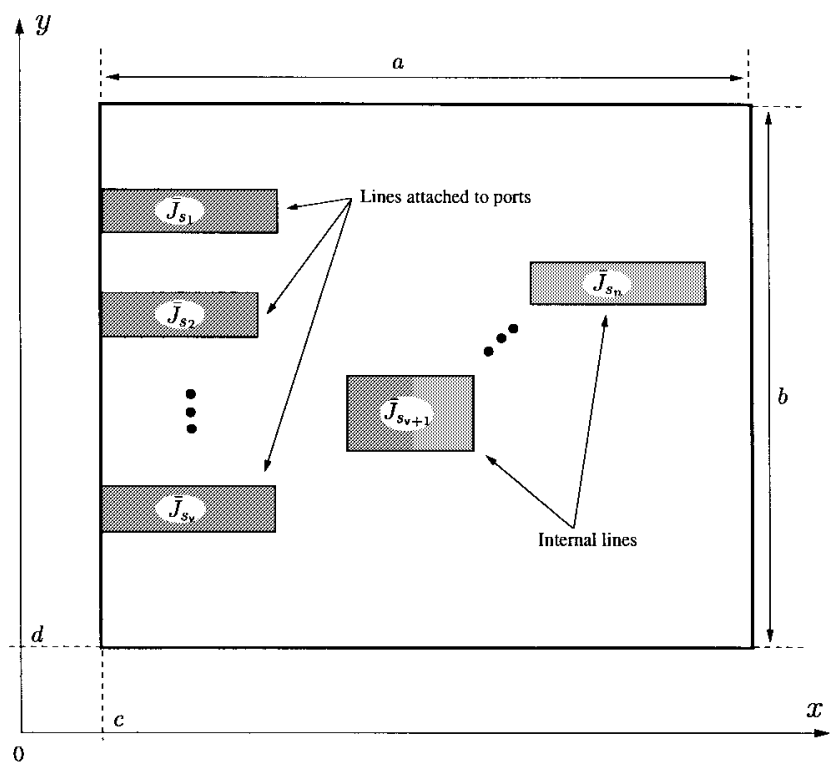

Fig. 2. General boxed printed circuit showing printed lines attached to ports and internal lines.

for the lines acting as ports and another for the other metallic areas, namely,

$$
\begin{aligned}
{\left.\left[\bar{E}_{t_{(e, q)}}+\bar{E}_{t_{(\mathrm{scat})}}\right]\right|_{s_{e_{q}}} } & =Z_{s} \bar{J}_{s_{q}}, & & 1 \leq q \leq v \\
\left.\bar{E}_{t_{(\mathrm{scat})}}\right|_{s_{e_{q}}} & =Z_{s} \bar{J}_{s_{q}}, & & v+1 \leq q \leq n
\end{aligned}
$$

Introducing the Green's function formalism, the total scattered field produced by the $n$-induced currents in the structure can be written by using the superposition principle as

$$
\bar{E}_{t_{(\text {scat }}}=\sum_{p=1}^{n} \int_{s_{e_{p}}} \overline{\bar{G}}_{E_{J}} \cdot \bar{J}_{s_{p}} d s^{\prime}
$$

and the dyadic electric-field Green's function $\overline{\bar{G}}_{E_{J}}$ can be expressed, in a boxed environment, with the following infinite modal sum:

$$
\begin{array}{r}
\overline{\bar{G}}_{E_{J}}\left(x, y, z, x^{\prime}, y^{\prime}, z^{\prime}\right) \\
=\sum_{m} V_{m}\left(z, z^{\prime}\right) \bar{e}_{m}(x, y) \bar{e}_{m}\left(x^{\prime}, y^{\prime}\right)
\end{array}
$$

where $\bar{e}_{m}$ are the orthonormal vector-mode functions used to represent the fields inside a waveguide as derived in [8], and the index $m$ covers all $\mathrm{TE}_{m, n}$ and $\mathrm{TM}_{m, n}$ modes of the box.

The voltage coefficient $V_{m}\left(z, z^{\prime}\right)$ (depending on the source-observer axial position) is computed using the transverse equivalent network shown in Fig. 3, which describes the longitudinal geometrical dependence of the structure [9], [10]. The voltages at all dielectric interfaces must be computed and, in Appendix I, an alternative procedure based on network calculations is described. After this calculation, (5) becomes the boxed Green's function including all layers.

An integral equation can now be formulated, including the presence of all of the layers and metallic elements. To do so, we simply enforce the boundary conditions for the transverse electric field at all metallic surfaces by introducing (4) in (3), so that

$$
\begin{gathered}
{\left.\left[\bar{E}_{t_{(e, q)}}=Z_{s} \bar{J}_{s_{q}}-\sum_{p=1}^{n} \int_{s_{e_{p}}} \overline{\bar{G}}_{E_{J}} \cdot \bar{J}_{s_{p}} d s^{\prime}\right]\right|_{s_{e_{q}}},} \\
1 \leq q \leq v \\
{\left.\left[0=Z_{s} \bar{J}_{s_{q}}-\sum_{p=1}^{n} \int_{s_{e_{p}}} \overline{\bar{G}}_{E_{J}} \cdot \bar{J}_{s_{p}} d s^{\prime}\right]\right|_{s_{e_{q}}},} \\
v+1 \leq q \leq n .
\end{gathered}
$$

This system of integral equations can now be solved with the method of moments (MoM). A key step in the solution 


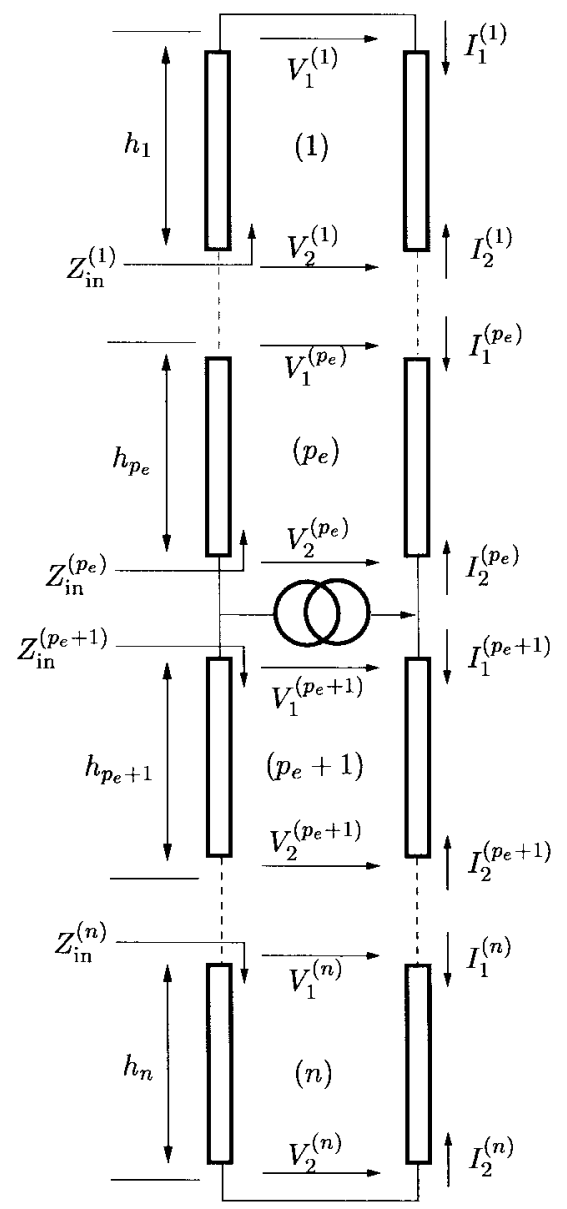

Fig. 3. Transverse transmission-line equivalent network representation for the structure in Fig. 1.

procedure is the proper choice of basis and test functions. A popular choice is to use the so-called subsectional rooftop functions, both as basis and test functions (Galerkin procedure). In this paper, we have instead used complete sets of entire-domain vector functions supported separately by each metallic area. These vector functions are essentially TE and TM vector-mode functions defined in each rectangular domain subject to appropriate boundary conditions.

There are two main advantages in using these basis (and test) functions in the MoM algorithm. First, the coefficient matrix elements of the MoM system obtained from the integral equations converges faster than the one obtained using subsectional rooftop functions. Second, the two-dimensional dependence of the currents on the metallic areas is naturally taken into account by the two-dimensional spatial dependence of the entire-domain vector basis functions. To better illustrate these points, we first show in Fig. 4 a typical relative error convergence plot when subsectional rooftop functions are used, as compared to what we obtain when the entire-domain functions are used. In the subsectional case, three cells were taken along the linewidth as required by the modeling of the transverse variation of the currents. Moreover, in the entiredomain case, the mode investigated is the $\mathrm{TM}_{2,0}$ mode of the new modal expansion set derived in Appendix II-A. The quantity plotted in Fig. 4 is the convergence relative error obtained in the sum of (5), after performing integration with the corresponding basis and test functions. From the relative errors obtained, a significantly faster numerical convergence is observable in the entire-domain case. In Fig. 5, we next show the transverse dependence of the two components of the current density induced on a printed line as obtained with the approach used in this paper. As can be seen, the results obtained show the singular behavior of the currents close to parallel edges.

Using then the entire-domain approach, all of the $n$ unknown currents induced on the metallic areas of the structure will take the following form:

$$
\bar{J}_{s_{p}}=\sum_{k} \alpha_{k}^{(p)} \bar{e}_{k}^{(p)}\left(x^{\prime}, y^{\prime}\right), \quad 1 \leq p \leq n
$$

where, again, the index $k$ covers all $\mathrm{TE}_{k, l}$ and $\mathrm{TM}_{k, l}$ modes.

It is important to observe that different modal expansions need to be used to represent the currents induced in different type of metallic areas. For instance, in the case of the metallic areas (or lines) connected to ports, the modal expansions need to be modified to account for the presence of the exciting electric field $\bar{E}_{t_{(e, q)}}$ of (6a). Furthermore, for lines with one side connected to the metallic box, the modal expansions are different to allow proper boundary conditions for the induced currents. The analytical expressions for all of the modal expansions required are given in Appendix II.

The application of the Galerkin formulation of the MoM to the system of integral equations shown in (6), with our selection of basis and test functions, leads to the following system of linear equations:

$$
\begin{array}{r}
P_{(e, q)}(i) \\
=Z_{s} \sum_{k} \alpha_{k}^{(q)} I_{e e}^{(q, q)}(i, k)+\sum_{p=1}^{n} \sum_{k} \alpha_{k}^{(p)} R^{(q, p)}(i, k), \\
1 \leq q \leq v
\end{array}
$$

where the matrix coefficients are given by the following integrals:

$$
\begin{aligned}
I_{e e}^{(q, q)}(i, k)= & +\int_{s_{e_{q}}} \bar{e}_{i}^{(q)}(x, y) \bar{e}_{k}^{(q)}(x, y) d s \\
R^{(q, p)}(i, k)=- & \int_{s_{e_{q}}} d s \bar{e}_{i}^{(q)}(x, y) \int_{s_{e_{p}}} d s^{\prime} \bar{e}_{k}^{(p)}\left(x^{\prime}, y^{\prime}\right) \\
& \cdot \overline{\bar{G}}_{E_{J}}
\end{aligned}
$$

and the known term vector of the system has been defined as

$$
P_{(e, q)}(i)=\int_{s_{e_{q}}} \bar{E}_{t_{(e, q)}} \bar{e}_{i}^{(q)}(x, y) d s
$$



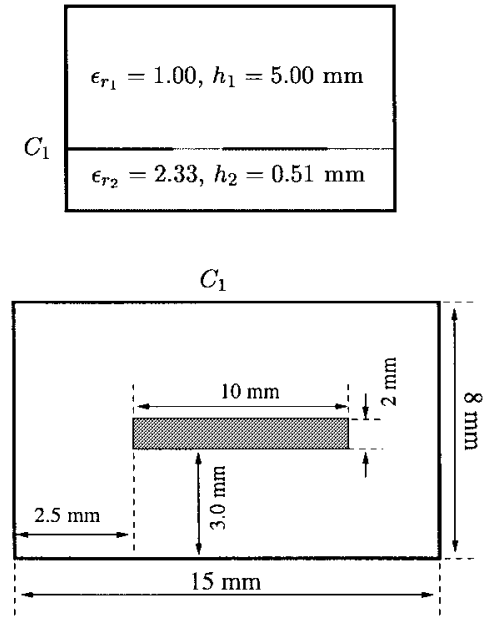

(a)

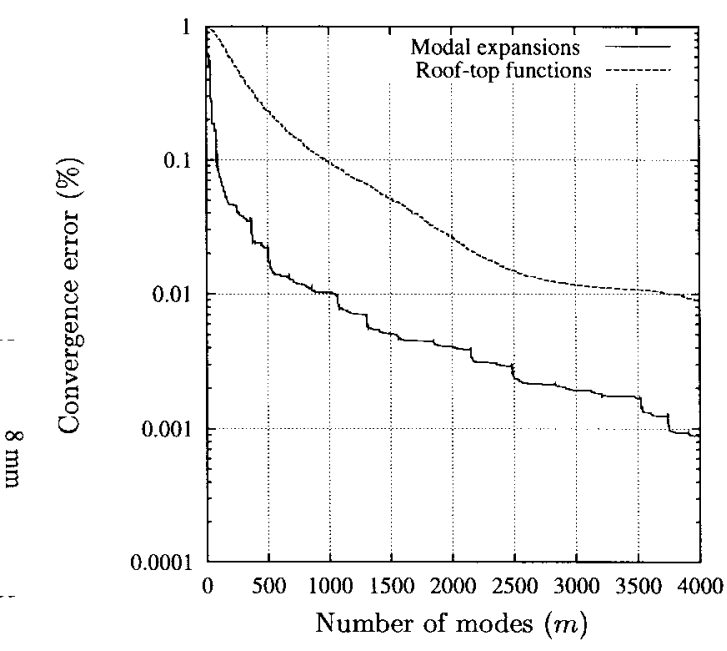

(b)

Fig. 4. Convergence relative errors obtained for the sum in (5) when modal expansions are used, and when rooftop functions are used as basis and test functions in the MoM algorithm. (a) Printed line under test. (b) Relative error.

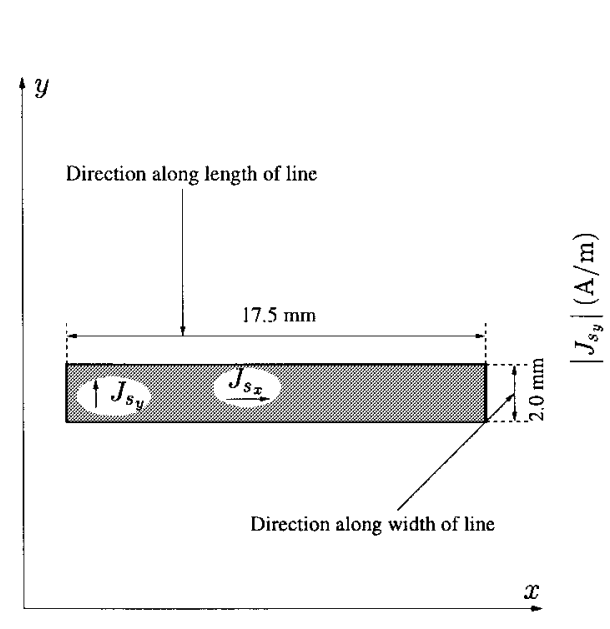

(a)

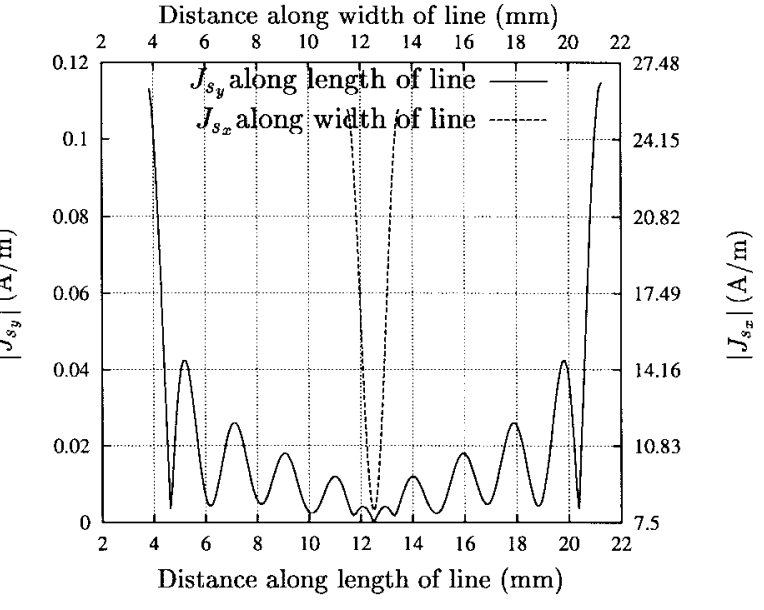

(b)

Fig. 5. Transverse dependence of the two components of the current density induced on a printed line showing the singular behavior close to parallel edges. Dimensions of the box: $a=25 \mathrm{~mm}, b=25 \mathrm{~mm}$. Substrate: $\epsilon_{r}=2.33, h=0.51 \mathrm{~mm}$. Frequency $5.9 \mathrm{GHz}$. (a) Metallic line under test. (b) Induced current density.

It is important to note that all of the above integrals can be easily solved analytically. The final structure of the system of linear equations to be inverted is shown in (11), at the bottom of this page.

It is worthwhile to mention here that, with our approach, the order of the linear system to be solved is $(n K)$, where $n$ is the number of lines and $K$ is the number of modal functions inside every line. Also, each matrix element calls for the evaluation of a modal sum that is expected to converge reasonably fast
(Fig. 4). Finally, it is important to point out that the technique is currently restricted to the analysis of nontouching metallic rectangles.

\section{EXCITATION AND PORT MOdeling}

One important aspect that deserves specific attention is the adaptation of the delta-gap excitation model described in [11], to the modal expansions used in this paper.

$$
\left(\begin{array}{c}
\underline{P}^{(e, 1)} \\
\vdots \\
\underline{P}^{(e, v)} \\
\underline{0} \\
\vdots \\
\underline{0}
\end{array}\right)=\left(\begin{array}{cccc}
{\left[Z_{s} \underline{\underline{I}}_{e e}^{(1,1)}+\underline{\underline{R}}^{(1,1)}\right]} & \underline{\underline{R}}^{(1,2)} & \cdots & \underline{\underline{R}}^{(1, n)} \\
\underline{\underline{R}}^{(2,1)} & {\left[Z_{s} \underline{\underline{I}}^{(2,2)}+\underline{\underline{R}}^{(2,2)}\right]} & \cdots & \underline{\underline{R}}^{(2, n)} \\
\vdots & \vdots & \ddots & \vdots \\
\underline{\underline{R}}^{(n, 1)} & \underline{\underline{R}}^{(n, 2)} & \cdots & {\left[Z_{s} \underline{\underline{I}}_{e e}^{(n, n)}+\underline{\underline{R}}^{(n, n)}\right.}
\end{array}\right) \cdot\left(\begin{array}{c}
\underline{\alpha}^{(1)} \\
\underline{\underline{\alpha}}^{(2)} \\
\vdots \\
\underline{\alpha}^{(n)}
\end{array}\right)
$$




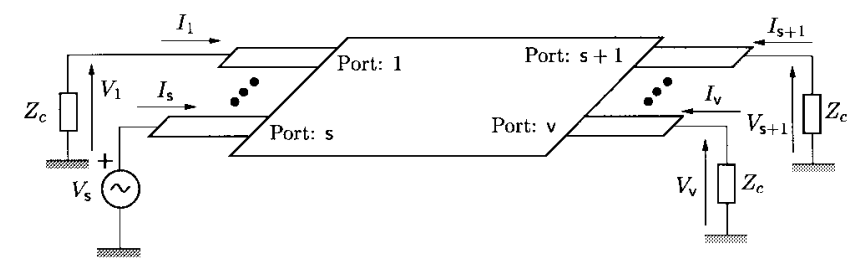

Fig. 6. Multiport network excited by a voltage generator at port $s$. All other ports are loaded with the characteristic impedance of reference.

Let us, for instance, consider the multiport network shown in Fig. 6. To extract the scattering parameters of this structure, we can place the exciting generator at a generic port $(s)$, as shown in Fig. 6, and load all other ports with the characteristic impedance $Z_{c}$. Following the delta-gap model [11], a voltage $V_{r}$ constant along the width of the line is assumed to be applied to each port. This is equivalent to taking the following dirac-delta expression for the exciting field $\bar{E}_{t_{(e, r)}}$ of (3a) at each port

$$
\bar{E}_{t_{(e, r)}}=-V_{r} \delta\left(x-x_{r}\right) \hat{u}_{x}, \quad 1 \leq r \leq v
$$

where $x_{r}$ is the position of the $r$ th port, $\hat{u}_{x}$ the unit vector along the $x$-axis of Fig. 6 , and only $x$-directed ports have been included in (12) to simplify notation. Also, to simplify notation, the pulse and delta functions along the $y$ - and $z$ directions, respectively, have been omitted, but it should be understood that the voltage source is explicitly restricted to the domain of the conductor, existing only along the width of the line to which the generator is applied.

For all ports connected to the characteristic impedance, the boundary condition imposed by the loads requires the following redefinition of the exciting electric field:

$$
\bar{E}_{t_{(e, r)}}=I_{r} Z_{c} \delta\left(x-x_{r}\right) \hat{u}_{x}, \quad 1 \leq r \leq v, \quad \text { for all } r \neq s
$$

and $I_{r}$ is the current flowing at the $r$ th port, as shown in Fig. 6 . This current can be easily computed by integrating the current density induced at the proper port position, namely,

$$
I_{r}=\int_{d(r)}^{d(r)+b(r)} \bar{J}_{s_{r}}\left(x_{r}, y^{\prime}\right) \cdot \hat{u}_{x} d y^{\prime}, \quad 1 \leq r \leq v
$$

where $d(r)$ and $b(r)$ are shown in Fig. 1 .

Using the expansion of the current density in (7), the total induced current at the $r$ th port is written as

$$
I_{r}=\sum_{k} \alpha_{k}^{(r)} \gamma_{k}^{(r)}, \quad 1 \leq r \leq v
$$

where the following coefficients have been defined:

$$
\gamma_{k}^{(r)}=\int_{d(r)}^{d(r)+b(r)} \bar{e}_{k}^{(r)}\left(x_{r}, y^{\prime}\right) \cdot \hat{u}_{x} d y^{\prime}, \quad 1 \leq r \leq v .
$$

The coefficients $\gamma$ defined above can be easily evaluated analytically. The explicit expressions required for all cases are given in Appendix III.
With the currents flowing at the ports expressed as in (15), the final exciting field in (13) for the ports connected to the loads can be written as

$$
\begin{array}{r}
\bar{E}_{t_{(e, r)}}=\sum_{k} \alpha_{k}^{(r)} \gamma_{k}^{(r)} Z_{c} \delta\left(x-x_{r}\right) \hat{u}_{x} ; \quad 1 \leq r \leq v, \\
\text { for all } r \neq s .
\end{array}
$$

Once an expression is available for the exciting electric field, we can compute the known term vectors of the MoM system associated with the ports connected to the loads. If the coefficients $\gamma$ defined in (16) are used again, the integral in (10) becomes

$$
\begin{aligned}
P_{(e, r)}(i)=\sum_{k} \alpha_{k}^{(r)} \gamma_{k}^{(r)} Z_{c} \gamma_{i}^{(r)} ; \quad 1 \leq r \leq v & \\
& \text { for all } r \neq s .
\end{aligned}
$$

The last vector that needs to be computed is the one associated with the port where the generator is placed. In this case, we take the applied voltage $V_{s}$ as the exciting voltage. Therefore, using (12) directly into (10), and using again the coefficients $\gamma$ defined in (16), one finally obtains

$$
P_{(e, s)}(i)=-V_{s} \gamma_{i}^{(s)} \text {. }
$$

The known term vectors associated with the ports connected to loads are expressed as a function of the unknown coefficients $\alpha_{k}^{(r)}$. As a consequence, these terms are moved to the right-hand side and are, therefore, absorbed in the system matrix. The true known term vector of the system is consequently composed only of the vector associated with the port where the exciting generator is placed (19). After all the required manipulations, the final MoM system becomes

$$
\underline{\underline{B}}=\underline{\underline{A}} \cdot \underline{\underline{X}}
$$

where

$$
\begin{aligned}
& \underline{\underline{B}} \\
& =\left(\begin{array}{c}
\underline{0} \\
\vdots \\
\underline{\gamma}^{(s)} \\
\vdots \\
\underline{0}
\end{array}\right) \\
& \left.\underline{\underline{X}} \begin{array}{c}
\frac{\alpha^{(1)}}{V_{s}} \\
\frac{\alpha^{(2)}}{V_{s}} \\
\vdots \\
\underline{\alpha}^{(s)} \\
V_{s} \\
\vdots \\
\frac{\underline{\alpha}^{(n)}}{V_{s}}
\end{array}\right)
\end{aligned}
$$

(21b) is shown at the bottom of the following page, all equations of the linear system have been divided by the 


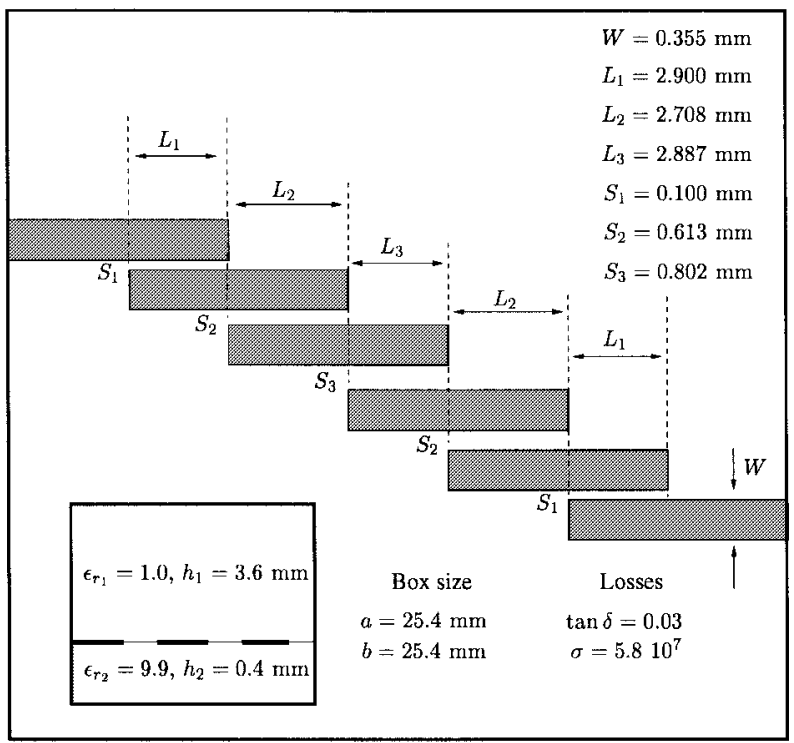

(a)

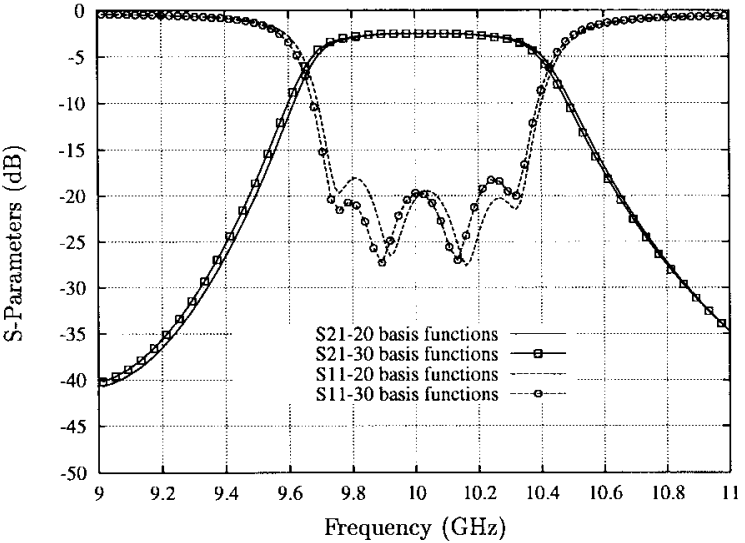

(b)

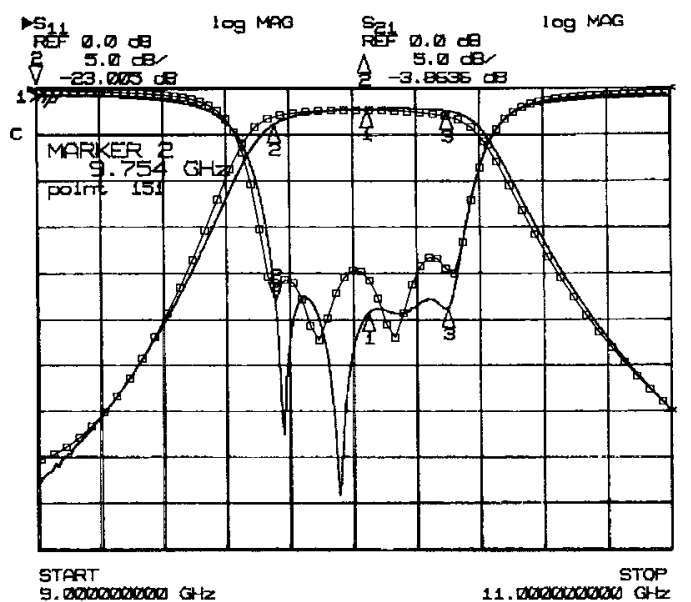

(c)

Fig. 7. Measured versus simulated results for the coupled-line bandpass filter shown. Convergence with the number of basis functions is also included. (a) Geometry of the filter. (b) Convergence study. (c) Measured versus simulated results. Solid line denotes measurements, while denote square points simulations.

exciting voltage $V_{s}$, and $\underline{\gamma}^{(r)^{T}}$ denotes the transpose of the corresponding $\gamma$ vector.

The input impedance can now be defined as the ratio between the applied voltage $V_{s}$ and induced current $I_{s}$ at the point of excitation. Using (7) and (14) for the computation of the current, and the definition in (16), the input admittance at the excitation port simply becomes

$$
Y_{\text {in }}=\frac{I_{s}}{V_{s}}=\sum_{k} \frac{\alpha_{k}^{(s)}}{V_{s}} \gamma_{k}^{(s)}
$$

$$
=\left(\begin{array}{cccccc}
{\left[Z_{s} \underline{\underline{I}}_{e e}^{(1)}+\underline{\underline{R}}^{(1,1)}-\underline{\gamma}^{(1)} Z_{c} \underline{\underline{\gamma}}^{(1)}\right]} & \underline{\underline{R}}^{(1,2)} & \ldots & \underline{\underline{R}}^{(1, s)} & \ldots & \underline{\underline{R}}^{(1, n)} \\
\underline{\underline{R}}^{(2,1)} & {\left[Z_{s} \underline{\underline{I}}_{e e}^{(2)}+\underline{\underline{R}}^{(2,2)}-\underline{\underline{\gamma}}^{(2)} Z_{c} \underline{\underline{\gamma}}^{(2)}\right]} & \ldots & \underline{\underline{R}}^{(2, s)} & \ldots & \underline{\underline{R}}^{(2, n)} \\
\vdots & \vdots & \ddots & \vdots & \ddots & \vdots \\
\underline{\underline{R}}^{(s, 1)} & \underline{\underline{R}}^{(s, 2)} & \ldots & {\left[Z_{s} \underline{\underline{I}}_{e e}^{(s)}+\underline{\underline{R}}^{(s, s)}\right]} & \ldots & \underline{\underline{\underline{R}}}^{(s, n)} \\
\underline{\underline{R}}^{(n, 1)} & \underline{\underline{R}}^{(n, 2)} & \ldots & \underline{\underline{R}}^{(n, s)} & \ldots & {\left[Z_{s} \underline{\underline{I}}^{(n)}+\underline{\underline{R}}^{(n, n)}\right]}
\end{array}\right)
$$




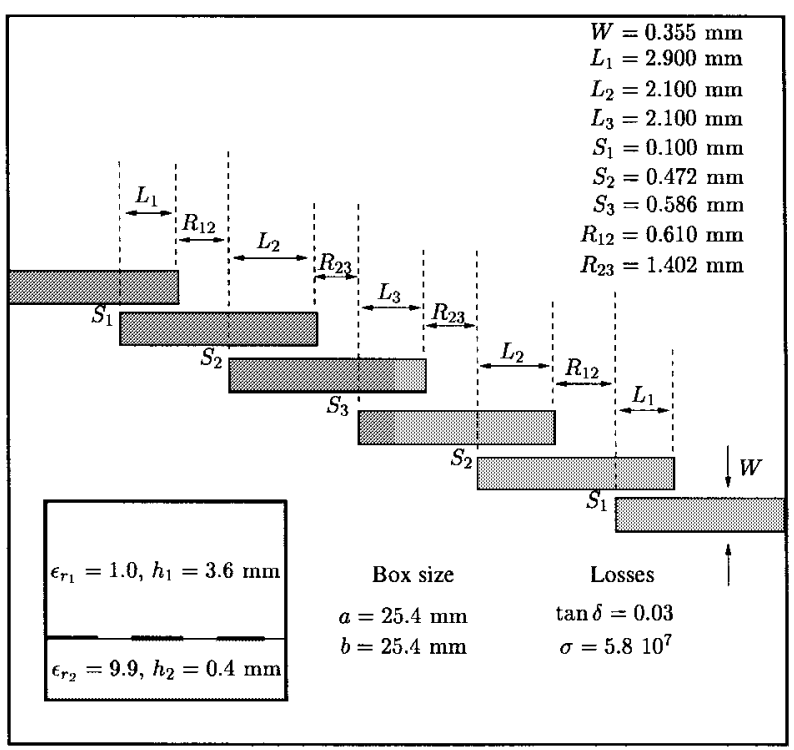

(a)

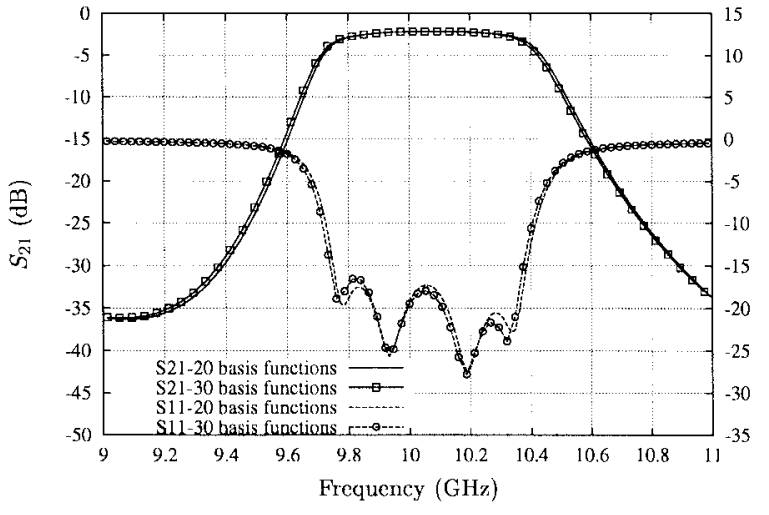

(b)

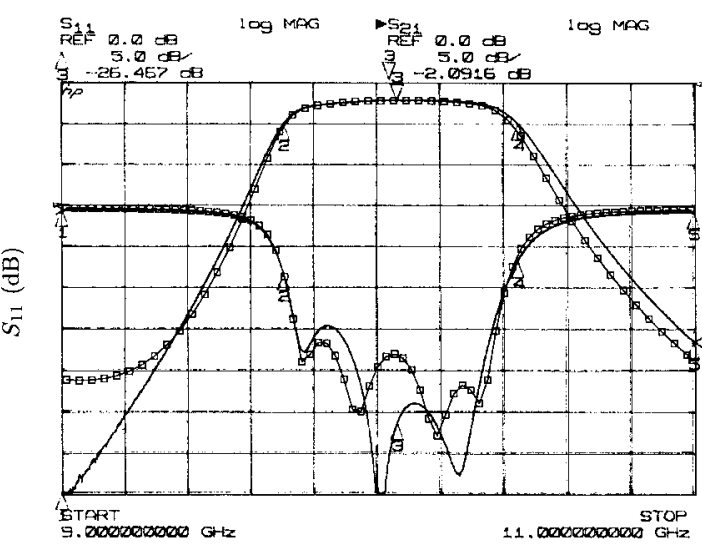

(c)

Fig. 8. Measured versus simulated results for the coupled-line bandpass filter shown. Convergence with the number of basis functions is also included. (a) Geometry of the filter. (b) Convergence study. (c) Measured versus simulated results. Solid line denotes measurements, while square points denote simulation.

The computation of the rest of the scattering matrix is accomplished by first evaluating all of the transadmittances, namely,

$$
Y_{\text {tran }}^{(r)}=\frac{I_{r}}{V_{s}}=\sum_{k} \frac{\alpha_{k}^{(r)}}{V_{s}} \gamma_{k}^{(r)}
$$

and then using standard network equations [12] so that

$$
S_{r, s}=\frac{2 Z_{c} Y_{\text {tran }}^{(r)}}{1+Z_{c} Y_{\mathrm{in}}^{-}}, \quad 1 \leq r \leq v, \quad \text { for all } r \neq s .
$$

The solution process just outlined is repeated by placing the exciting generator at all ports consecutively $(1 \leq s \leq v)$, thus evaluating all of the $S$-parameters of the structure.

\section{RESULTS}

To further increase the computational efficiency of the theory described in this paper, the asymptotic term of the kernel of the system of integral equations has been extracted following a technique similar to the one described in [7].
To validate the computer-aided design (CAD) tool obtained, the two bandpass filter examples reported in [13] have been analyzed, and the results obtained have been compared with measurements on actual hardware. Fig. 7 shows the results obtained for a bandpass filter based on coupled lines sections. As we see, the agreement between computed and measured results is, in general, good. Also, Fig. 7(b) presents the results when 20 and 30 modes are used in the analysis, indicating that convergence is attained rapidly since only small variations in the final results are observed.

Fig. 8 presents another bandpass filter in which the coupledline sections are connected via a small portion of microstrip transmission line. As we see also in this case, the agreement between computed and measured results is good. Again, Fig. 8(b) presents the results when 20 and 30 modes are used in the analysis, showing that convergence is also good since only small changes in the final computed responses are obtained.

For these two examples, a total of 18000 modes were used in the summation of the frequency-independent part of 


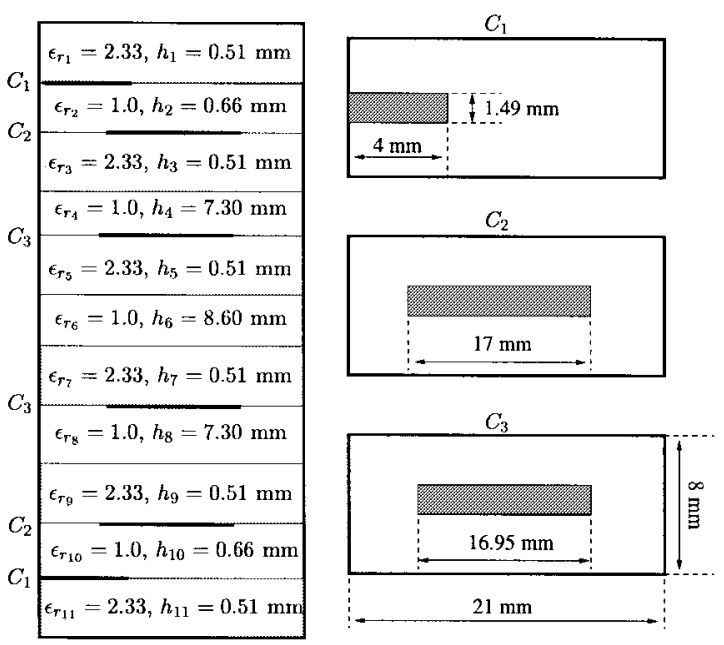

(a)

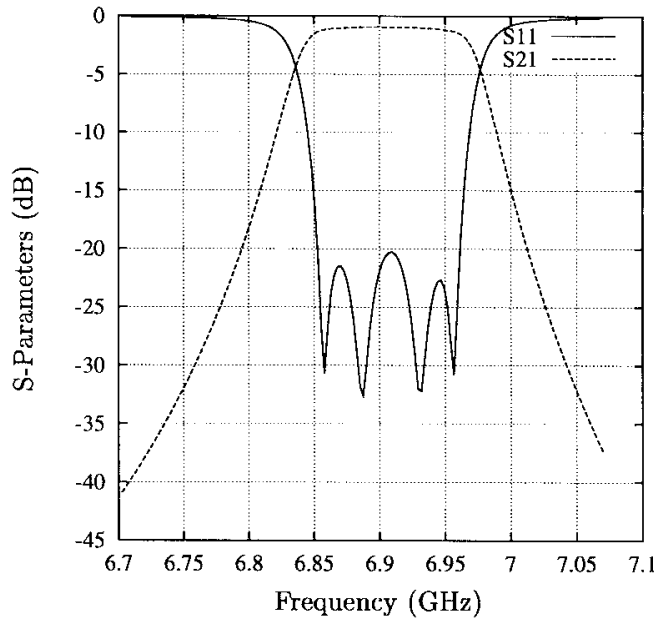

(b)

Fig. 9. Response of the broadside-coupled filter after optimization. (a) Geometry of the filter. (b) Electrical response.

the kernel, while only 2000 modes were retained for the frequency-dependent part. The corresponding central processing unit (CPU) times on a HP712/80 workstation are 3 min for the initial frequency-independent calculation followed by $6 \mathrm{~s}$ for each frequency point. This performance represents a considerable reduction in computational time with respect to the data reported in [7].

\section{APPLicAtions}

The software tool described in this paper is computationally very efficient so that it can be effectively used as an interactive CAD tool for the design of complex multilayer circuits such as microwave filters [14], [15]. As an application example, we will now describe a number of practical filter structures that have been optimized with the CAD tool developed.

\section{A. Broadside-Coupled Filters}

A common implementation of microwave filters in planar technology is to use side-coupled transmission-line sections. An alternative implementation is the broadside-coupled configuration. Fig. 9 shows a broadside-coupled filter in which all resonators are printed on different substrates and placed one above the other. As we can observe from Fig. 9, the broadside configuration is more compact, thus resulting in a considerable reduction in the total size of the filter. In addition, the coupling between resonators can be substantially increased, thus avoiding the need for very narrow gaps between lines, as usually required in wide-band side-coupled structure.

\section{B. Implementing Transmission Zeros}

It is well known that the implementation of transmission zeros in the insertion loss response of microwave filters can be obtained by introducing coupling between nonadjacent resonators (cross coupling) [16]. A very compact implementation of transmission zeros in printed structures can be obtained if the resonators of a microwave filter are printed on different substrates. In this way, cross coupling between nonadjacent resonators can be easily introduced in addition to the normal signal path of the filter. In general, a coupling between resonators 1 and 3 gives a single zero below or above the passband, depending on the sign of the coupling. In Fig. 10(a), we present a simple three-layered printed structure that implements a single transmission zero above the passband of the filter following the 1-3 cross-coupling concept. In order to easily realize this structure, the substrates used to print the resonators are separated by a foam of dielectric constant close to unity $\left(\epsilon_{r}=1.07\right)$. This structure has been manufactured and tested and, in Fig. 10(c), we present the simulated versus measured results. As we can observe, the agreement with simulated results is, in general, good, and the position of the implemented transmission zero is close to the expected computed location. Moreover, Fig. 10(b) presents the simulated results when 20 and 30 modes are included in the computations. Good numerical convergence is achieved, and only minor differences in the final results are observed between the two calculated responses.

Due to the high saturation level of the frequency-band spectrum, there are many situations in which high selectivity is required on both sides of the passband. The previous 1-3 cross-coupling configuration can also be used for this purpose, together with an additional resonator acting as an "extracted pole" [17] to introduce a transmission zero on the lower side of the passband. Following this concept, a filter structure has been optimized so that both transmission zeros are symmetrically disposed around the useful passband. In Fig. 11, we present both the final structure and simulated electrical response. It is interesting to remark that a pair of transmission zeros can also be implemented introducing a single cross coupling between resonators 1 and 4 of a fourth-order configuration when the cross coupling is of a sign opposite the main signal coupling.

\section{CONCLUSION}

An accurate and efficient CAD tool for the analysis of a wide class of multilayer boxed printed circuits has been 


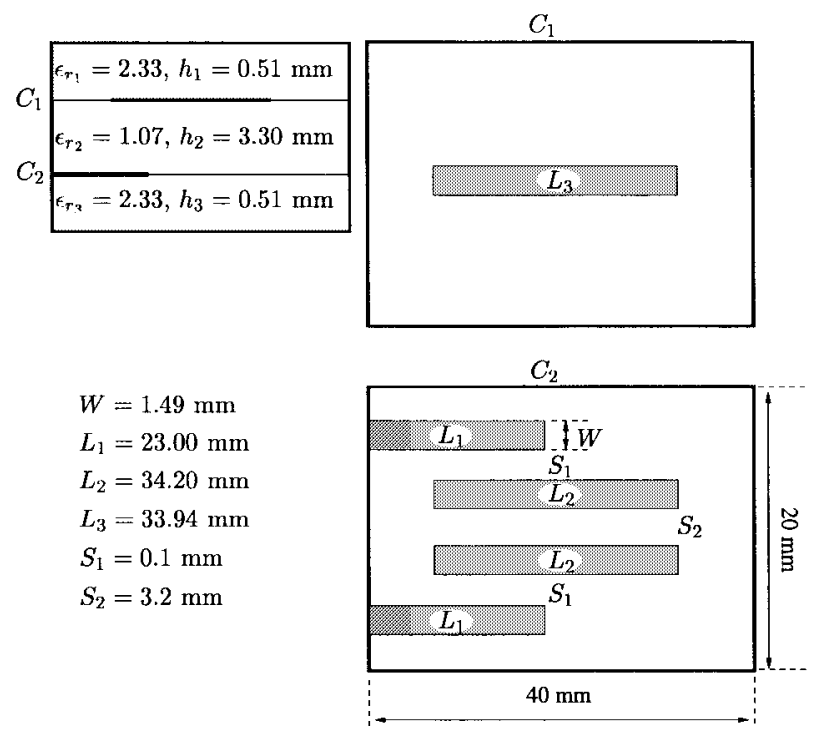

(a)

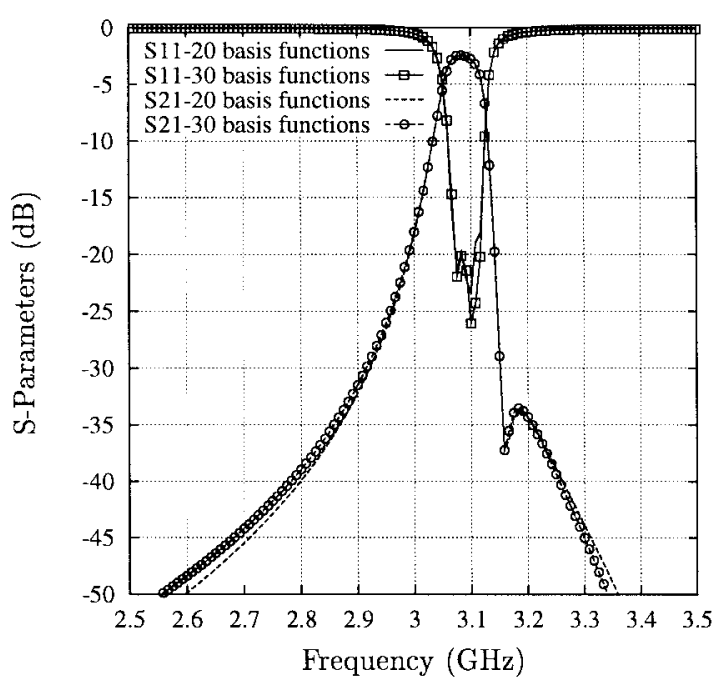

(b)

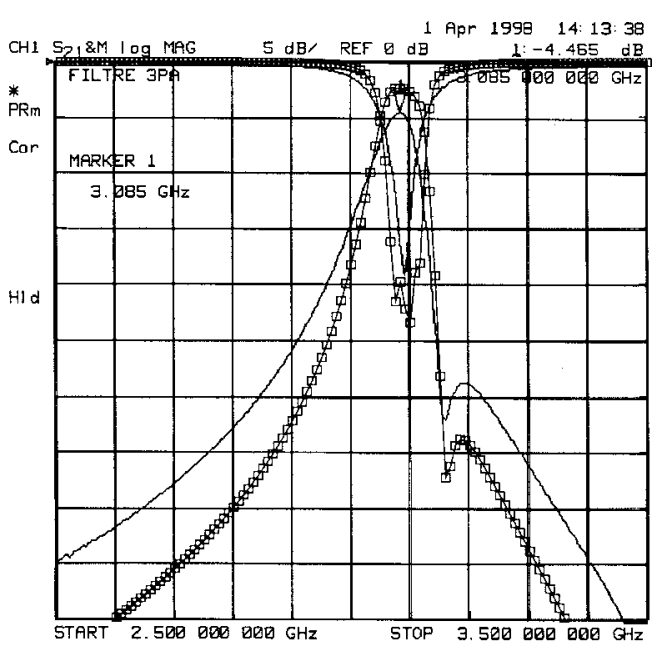

(c)

Fig. 10. Filter with transmission zero above the passband due to cross coupling between first and third resonators. (a) Geometry of the filter. (b) Convergence study. (c) Measured versus simulated results. Solid line denotes measurements, while square points denote simulations.

described in this paper. The tool is based on an integralequation formulation, which calls for the evaluation of the multilayer space-domain boxed Green's functions. The boxed Green's functions have been expressed in a very compact form using a modal expansion notation. Transmission-line concepts have also been used to account for an arbitrary number of dielectric layers. Furthermore, the integral equation is solved using special entire-domain basis and test vector functions in order to enhance the computational efficiency. Finally, the traditional delta-gap concept used to model the excitation has been reformulated to account for the vector expansions used.

In addition to theory, simulated and measured results for practical microwave filters have also been compared, showing that the CAD tool developed is indeed accurate and efficient. The usefulness and versatility of the CAD tool developed has also been illustrated by investigating several practical filter structures including broadside-coupled filters, and high-selectivity filters exhibiting transmission zeros near their passband response.

\section{APPENDIX I VOLTAGE COMPUTATION}

It is well known that the longitudinal dependence, along the stratification axis, of a multilayered printed structure can be reduced to the evaluation of the voltages and currents on an equivalent transmission-line network [9], [10]. For the general structure shown in Fig. 1, the voltage coefficients of the Green's functions in (5) can, therefore, be obtained from the network of Fig. 3. The computations can be easily performed by first replacing each transmission line section of length $l_{i}$ by an equivalent two-port impedance network 


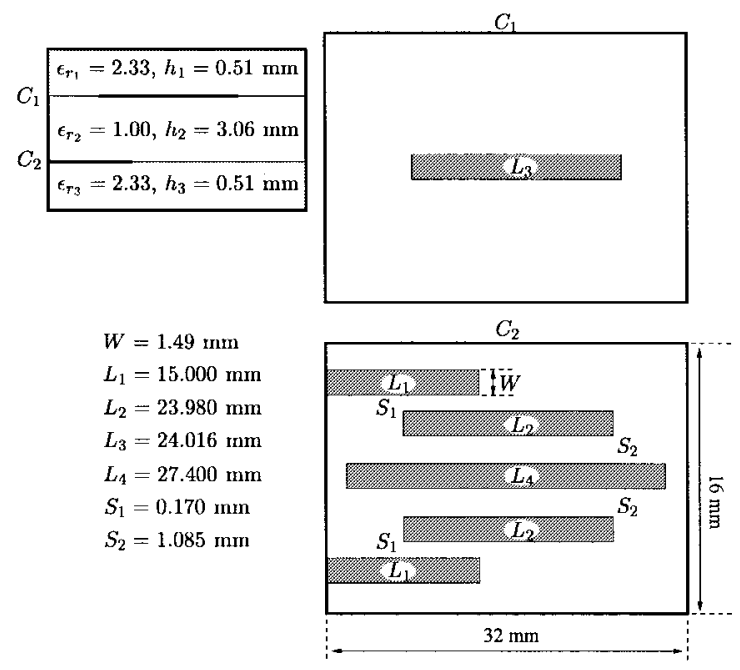

(a)

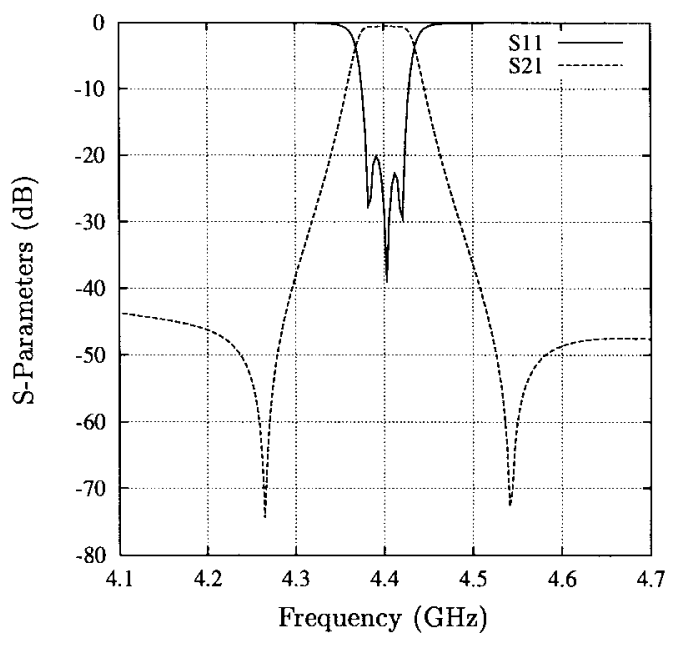

(b)

Fig. 11. Filter with transmission zeros on both sides of the useful passband. Transmission zero below the passband is due to an extracted pole implemented by line $L_{4}$. (a) Geometry of the filter. (b) Electrical response.

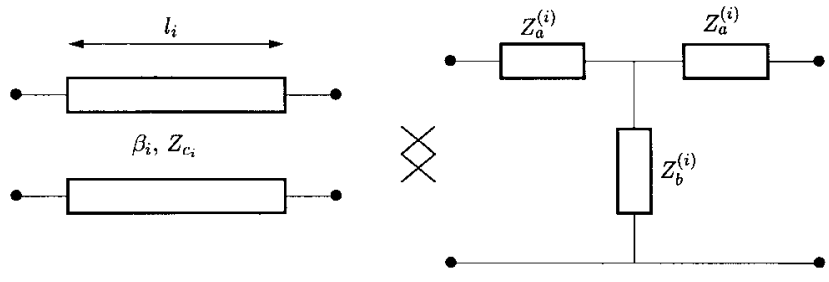

Fig. 12. Equivalent impedance network of a length of transmission line.

(Fig. 12)

$$
\begin{aligned}
& Z_{a}^{(i)}=+j Z_{c_{i}} \tan \left(\beta_{i} \frac{l_{i}}{2}\right) \quad Z_{11}^{(i)}=Z_{22}^{(i)}=Z_{a}^{(i)}+Z_{b}^{(i)} \\
& Z_{b}^{(i)}=-j Z_{c_{i}}, \csc \left(\beta_{i} l_{i}\right) \quad Z_{12}^{(i)}=Z_{21}^{(i)}=Z_{b}^{(i)}
\end{aligned}
$$

where $\beta_{i}$ is the propagation constant of the $i$ th transmission line and $Z_{c_{i}}$ its characteristic impedance. By now performing simple network operations, all of the electrical parameters of the circuit in Fig. 3 can be computed with the following iterative scheme.

First, compute the input impedance at the first interface

$$
Z_{\text {in }}^{(1)}=j Z_{c_{1}} \tan \left(\beta_{1} h_{1}\right)
$$

and then compute all other input impedances up to the position of the generator

$$
Z_{\mathrm{in}}^{(i)}=Z_{c_{i}} \frac{Z_{\mathrm{in}}^{(i-1)}+j Z_{c_{i}} \tan \left(\beta_{i} h_{i}\right)}{Z_{c_{i}}+j Z_{\mathrm{in}}^{(i-1)} \tan \left(\beta_{i} h_{i}\right)}, \quad i=2,3, \cdots, p_{e} .
$$

Next, compute the input impedance at the last interface

$$
Z_{\mathrm{in}}^{(n)}=j Z_{c_{n}} \tan \left(\beta_{n} h_{n}\right)
$$

finally, evaluate all other input impedances below the generator

$$
\begin{aligned}
Z_{\mathrm{in}}^{(i)}=Z_{c_{i}} \frac{Z_{\mathrm{in}}^{(i+1)}+j Z_{c_{i}} \tan \left(\beta_{i} h_{i}\right)}{Z_{c_{i}}+j Z_{\mathrm{in}}^{(i+1)} \tan \left(\beta_{i} h_{i}\right)}, \\
\quad i=n-1, n-2, \cdots, p_{e}+1 .
\end{aligned}
$$

With the above expression, it is now easy to compute the voltages and currents at the generator terminals, namely,

$$
\begin{gathered}
V_{2}^{\left(p_{e}\right)}=V_{1}^{\left(p_{e}+1\right)}=\frac{Z_{\mathrm{in}}^{\left(p_{e}\right)} Z_{\mathrm{in}}^{\left(p_{e}+1\right)}}{Z_{\mathrm{in}}^{\left(p_{e}\right)}+Z_{\mathrm{in}}^{\left(p_{e}+1\right)}} \\
I_{2}^{\left(p_{e}\right)}=\frac{V_{2}\left(p_{e}\right)}{Z_{\mathrm{in}}^{\left(p_{e}\right)}}=\frac{Z_{\mathrm{in}}^{\left(p_{e}+1\right)}}{Z_{\mathrm{in}}^{\left(p_{e}\right)}+Z_{\mathrm{in}}^{\left(p_{e}+1\right)}}, \\
I_{1}^{\left(p_{e}+1\right)}=\frac{V_{1}^{\left(p_{e}+1\right)}}{Z_{\mathrm{in}}^{\left(p_{e}+1\right)}}=\frac{Z_{\mathrm{in}}^{\left(p_{e}\right)}}{Z_{\mathrm{in}}^{\left(p_{e}\right)}+Z_{\mathrm{in}}^{\left(p_{e}+1\right)}}
\end{gathered}
$$

so that all other voltages and currents above the generator become

$$
\begin{array}{r}
I_{1}^{(i)}=\frac{V_{2}^{(i)}-Z_{22}^{(i)} I_{2}^{(i)}}{Z_{21}^{(i)}} \quad V_{2}^{(i-1)}=+V_{1}^{(i)} \\
V_{1}^{(i)}=Z_{11}^{(i)} I_{1}^{(i)}+Z_{12}^{(i)} I_{2}^{(i)} \quad I_{2}^{(i-1)}=-I_{1}^{(i)} \\
\quad i=p_{e}, p_{e}-1, \cdots, 2 .
\end{array}
$$

Finally, the voltages and currents in all interfaces below the generator are also computed as

$$
\begin{gathered}
I_{2}^{(i)}=\frac{V_{1}^{(i)}-Z_{11}^{(i)} I_{1}^{(i)}}{Z_{12}^{(i)}} \quad V_{1}^{(i+1)}=+V_{2}^{(i)} \\
V_{2}^{(i)}=Z_{21}^{(i)} I_{1}^{(i)}+Z_{22}^{(i)} I_{2}^{(i)} \quad I_{1}^{(i+1)}=-I_{2}^{(i)}, \\
\quad i=p_{e}+1, p_{e}+2, \cdots, n-1
\end{gathered}
$$

with $p_{e}$ denoting the order of the layer above the position of the generator, as shown in Fig. 3. 


\section{APPENDIX II \\ VECTOR EXPANSION FUNCTIONS}

The integral equation formulated in this paper is solved using special vector expansion functions, which are suitable to represent the currents induced on each metallic area. The functions are different according to the characteristics of each area of the circuit, so that the local boundary conditions for the induced currents are respected. In this appendix, we give the explicit expressions for all required functions. It is important to notice that the vector modal functions derived next are used in (7) to represent the unknown induced currents at all metallic areas of the structure.

\section{A. Internal Areas}

Expansion functions, which are suitable for representing the electric currents induced on a metallic area internal to the boxed circuit of Fig. 1, can be easily obtained by considering the modes of a waveguide bounded by four magnetic walls. Using the notation introduced in [8] and using the duality principle, we can directly write for a waveguide with magnetic walls

$$
\begin{aligned}
& e_{x_{m, n}}^{\xi}(x, y) \\
& =N_{e_{x}}^{\xi} \sin \left[\frac{m \pi}{a(p)}(x-c(p))\right] \cos \left[\frac{n \pi}{b(p)}(y-d(p))\right] \\
& e_{y_{m, n}}^{\xi}(x, y) \\
& =N_{e_{y}}^{\xi} \cos \left[\frac{m \pi}{a(p)}(x-c(p))\right] \sin \left[\frac{n \pi}{b(p)}(y-d(p))\right]
\end{aligned}
$$

where $\xi$ indicates $\mathrm{TE}_{m, n}$ or $\mathrm{TM}_{m, n}$ modes, $a(p)$ and $b(p)$ are the dimensions of the $p$ th rectangular area, and $c(p)$ and $d(p)$ are the offsets with respect the origin of coordinates as sketched in Fig. 1. The normalization factors for both families of modes are given by

$$
\begin{aligned}
& N_{e_{x}}^{\mathrm{TM}}=+\frac{\sqrt{\epsilon_{m} \epsilon_{n}}}{a(p)} \frac{m}{\sqrt{m^{2} \frac{b(p)}{a(p)}+n^{2} \frac{a(p)}{b(p)}}} \\
& N_{e_{y}}^{\mathrm{TM}}=+\frac{\sqrt{\epsilon_{m} \epsilon_{n}}}{b(p)} \frac{n}{\sqrt{m^{2} \frac{b(p)}{a(p)}+n^{2} \frac{a(p)}{b(p)}}}, \\
& m=0,1,2,3, \cdots ; \quad n=0,1,2,3, \cdots ; \\
& m=n \neq 0 \text {. } \\
& N_{e_{x}}^{\mathrm{TE}}=-\frac{2}{b(p)} \frac{n}{\sqrt{m^{2} \frac{b(p)}{a(p)}+n^{2} \frac{a(p)}{b(p)}}} \\
& N_{e_{y}}^{\mathrm{TE}}=+\frac{2}{a(p)} \frac{m}{\sqrt{m^{2} \frac{b(p)}{a(p)}+n^{2} \frac{a(p)}{b(p)}}} \\
& m=1,2,3, \cdots ; \quad n=1,2,3, \cdots
\end{aligned}
$$

where the constants $\epsilon_{m}$ and $\epsilon_{n}$ have been defined as [8]

$$
\epsilon_{m}= \begin{cases}1, & \text { if } m=0 \\ 2, & \text { if } m \neq 0\end{cases}
$$

\section{B. Lines Attached to $x=0$ Wall}

Similar to what was done in the previous section, we can now treat the case of an area connected electrically to the metallic wall $x=0$ of the box, as shown in Fig. 1. This situation arises when modeling a resonator connected to the box or when modeling input and output ports. In the case of a port, the expansion functions need to be modified to allow for the existence of an appropriate exciting electric field at the port position. While, if a resonator is connected to the wall, the expansion functions must satisfy the proper boundary conditions for the induced currents.

It can be easily shown that a suitable expansion set is generated by considering a waveguide with three magnetic walls as before, and one electric wall for the side connected to the box. The vector-mode functions can then be derived from the following scalar potentials, which respect the proper boundary conditions at the waveguide walls (electric and magnetics):

$$
\begin{aligned}
& \Phi_{m, n}^{\mathrm{TM}} \\
& \begin{aligned}
&=\frac{\sqrt{2 \epsilon_{n}}}{\pi} \frac{1}{\sqrt{m^{2} \frac{b(p)}{4 a(p)}+\mathrm{n}^{2} \frac{a(p)}{b(p)}}} \sin \left[\frac{m \pi}{2 a(p)}(x-c(p))\right] \\
& \cdot \cos \left[\frac{n \pi}{b(p)}(y-d(p))\right], m=1,3,5, \cdots \text { (odd); } \\
& n=0,1,2, \cdots
\end{aligned} \\
& \Phi_{m, n}^{\mathrm{TE}} \\
& \begin{array}{c}
=\frac{2}{\pi} \frac{1}{\sqrt{m^{2} \frac{b(p)}{4 a(p)}+n^{2} \frac{a(p)}{b(p)}}} \cos \left[\frac{m \pi}{2 a(p)}(x-c(p))\right] \\
\cdot \sin \left[\frac{n \pi}{b(p)}(y-d(p))\right], \\
m=1,3,5, \cdots(\text { odd }) \\
n=1,2,3, \cdots
\end{array}
\end{aligned}
$$

Using the above potentials, the vector-mode functions become

$$
\begin{aligned}
& e_{x_{m, n}}^{\xi}(x, y) \\
& \quad=N_{e_{x}}^{\xi} \cos \left[\frac{m \pi}{2 a(p)}(x-c(p))\right] \cos \left[\frac{n \pi}{b(p)}(y-d(p))\right] \\
& e_{y_{m, n}}^{\xi}(x, y) \\
& \quad=N_{e_{y}}^{\xi} \sin \left[\frac{m \pi}{2 a(p)}(x-c(p))\right] \sin \left[\frac{n \pi}{b(p)}(y-d(p))\right]
\end{aligned}
$$


and the normalization factors now take the following values:

$$
\begin{aligned}
& N_{e_{x}}^{\mathrm{TM}}=-\frac{\sqrt{2 \epsilon_{n}}}{2 a(p)} \frac{m}{\sqrt{m^{2} \frac{b(p)}{4 a(p)}+n^{2} \frac{a(p)}{b(p)}}} \\
& N_{e_{y}}^{\mathrm{TM}}=+\frac{\sqrt{2 \epsilon_{n}}}{b(p)} \frac{n}{\sqrt{m^{2} \frac{b(p)}{4 a(p)}+n^{2} \frac{a(p)}{b(p)}}}, \\
& N_{e_{x}}^{\mathrm{TE}}=-\frac{2}{b(p)} \frac{n}{n}=0,1,2, \cdots \\
& N_{e_{y}}^{\mathrm{TE}}=-\frac{2}{2 a(p)} \frac{n}{\sqrt{m^{2} \frac{b(p)}{4 a(p)}+n^{2} \frac{a(p)}{b(p)}}} \quad(38 \mathrm{~b}) ; \\
& \sqrt{m^{2} \frac{b(p)}{4 a(p)}+n^{2} \frac{a(p)}{b(p)}}
\end{aligned}
$$

where, again, $a(p), b(p)$ are the dimensions of the $p$ th rectangular area and $c(p), d(p)$ are the offsets with respect to the origin of coordinates, as shown in Fig. 1.

\section{Lines Attached to $x=a$ Wall}

Similar considerations apply for areas connected to the wall at $x=a$, as shown in Fig. 1 . The potentials respecting the proper boundary conditions take the form

$$
\begin{aligned}
& \Phi_{m, n}^{\mathrm{TM}}= \frac{\sqrt{2 \epsilon_{n}}}{\pi} \frac{1}{\sqrt{m^{2} \frac{b(p)}{4 a(p)}+n^{2} \frac{a(p)}{b(p)}}} \\
& \cdot \cos \left[\frac{m \pi}{2 a(p)}(x-c(p))\right] \cos \left[\frac{n \pi}{b(p)}(y-d(p))\right], \\
& m=1,3,5, \cdots \text { (odd); } \\
& n=0,1,2, \cdots, \quad \text { (39a) } \\
& \Phi_{m, n}^{\mathrm{TE}}= \frac{2}{\pi} \frac{1}{\sqrt{m^{2} \frac{b(p)}{4 a(p)}+n^{2} \frac{a(p)}{b(p)}}} \sin \left[\frac{m \pi}{2 a(p)}(x-c(p))\right] \\
& \cdot \sin \left[\frac{n \pi}{b(p)}(y-d(p))\right], \quad m=1,3,5, \cdots \text { (odd); } \\
& \quad n=1,2,3, \cdots .
\end{aligned}
$$

Vector-mode functions are now derived from the above potentials obtaining

$$
\begin{aligned}
& e_{x_{m, n}}^{\xi}(x, y) \\
& \quad=N_{e_{x}}^{\xi} \sin \left[\frac{m \pi}{2 a(p)}(x-c(p))\right] \cos \left[\frac{n \pi}{b(p)}(y-d(p))\right] \\
& e_{y_{m, n}}^{\xi}(x, y) \\
& \quad=N_{e_{y}}^{\xi} \cos \left[\frac{m \pi}{2 a(p)}(x-c(p))\right] \sin \left[\frac{n \pi}{b(p)}(y-d(p))\right] .
\end{aligned}
$$

The normalization factors finally take the form

$$
\begin{aligned}
& N_{e_{x}}^{\mathrm{TM}}=+\frac{\sqrt{2 \epsilon_{n}}}{2 a(p)} \frac{m}{\sqrt{m^{2} \frac{b(p)}{4 a(p)}+n^{2} \frac{a(p)}{b(p)}}} \\
& N_{e_{y}}^{\mathrm{TM}}=+\frac{\sqrt{2 \epsilon_{n}}}{b(p)} \frac{n}{\sqrt{m^{2} \frac{b(p)}{4 a(p)}+n^{2} \frac{a(p)}{b(p)}}}, \\
& m=1,3, \cdots \text { (odd); } \\
& n=0,1,2, \cdots \\
& N_{e_{x}}^{\mathrm{TE}}=-\frac{2}{b(p)} \frac{n}{\sqrt{m^{2} \frac{b(p)}{4 a(p)}+n^{2} \frac{a(p)}{b(p)}}} \\
& N_{e_{y}}^{\mathrm{TE}}=+\frac{2}{2 a(p)} \frac{m}{\sqrt{m^{2} \frac{b(p)}{4 a(p)}+n^{2} \frac{a(p)}{b(p)}}}, \\
& m=1,3, \cdots \text { (odd); } \\
& n=1,2,3, \cdots \text {. }
\end{aligned}
$$

\section{Lines Attached to $y=0$ or $y=b$ Walls}

Due to the separability of Maxwell's equations in the geometry of interest, lines attached to the walls $y=0$ and $y=b$ of Fig. 1 can simply be treated by changing, in the above expressions, all quantities related to the $x$-axis with the corresponding quantities related to the $y$-axis. This is also applicable to $y$-directed ports or when a resonator is electrically connected to other walls. For the sake of completeness, we also list here the analytic expressions of the modal expansions needed to characterize lines attached to walls at $y=0$ and $y=b$.

For a line attached to the wall $y=0$ of Fig. 1, we write from (37) and (38),

$$
\begin{aligned}
& e_{x_{m, n}}^{\xi}(x, y) \\
& \quad=N_{e_{x}}^{\xi} \sin \left[\frac{m \pi}{a(p)}(x-c(p))\right] \sin \left[\frac{n \pi}{2 b(p)}(y-d(p))\right] \\
& e_{y_{m, n}}^{\xi}(x, y) \\
& \quad=N_{e_{y}}^{\xi} \cos \left[\frac{m \pi}{a(p)}(x-c(p))\right] \cos \left[\frac{n \pi}{2 b(p)}(y-d(p))\right]
\end{aligned}
$$

$$
\begin{aligned}
& N_{e_{x}}^{\mathrm{TM}}=+\frac{\sqrt{\epsilon_{m}^{2}}}{a(p)} \frac{m}{\sqrt{m^{2} \frac{b(p)}{a(p)}+n^{2} \frac{a(p)}{4 b(p)}}} \\
& N_{e_{y}}^{\mathrm{TM}}=-\frac{\sqrt{\epsilon_{m} 2}}{2 b(p)} \frac{n}{\sqrt{m^{2} \frac{b(p)}{a(p)}+n^{2} \frac{a(p)}{4 b(p)}}},
\end{aligned}
$$

$$
\begin{aligned}
m & =0,1,2, \cdots ; \\
n & =1,3, \cdots \text { (odd). }
\end{aligned}
$$




$$
\begin{aligned}
N_{e_{x}}^{\mathrm{TE}}=-\frac{2}{2 b(p)} \frac{n}{\sqrt{m^{2} \frac{b(p)}{a(p)}+n^{2} \frac{a(p)}{4 b(p)}}} & \\
N_{e_{y}}^{\mathrm{TE}}=-\frac{2}{a(p)} \frac{m}{\sqrt{m^{2} \frac{b(p)}{a(p)}+n^{2} \frac{a(p)}{4 b(p)}}}, & \\
& m=1,2,3, \cdots ; \\
n & =1,3, \cdots \text { (odd). }
\end{aligned}
$$

Finally, for a line attached to the wall $y=b$ of Fig. 1 , we write instead from (40) and (41),

$$
\begin{aligned}
& e_{x_{m, n}}^{\xi}(x, y) \\
& =N_{e_{x}}^{\xi} \sin \left[\frac{m \pi}{a(p)}(x-c(p))\right] \sin \left[\frac{n \pi}{2 b(p)}(y-d(p))\right] \\
& e_{y_{m, n}}^{\xi}(x, y) \\
& =N_{e_{y}}^{\xi} \cos \left[\frac{m \pi}{a(p)}(x-c(p))\right] \cos \left[\frac{n \pi}{2 b(p)}(y-d(p))\right] \\
& N_{e_{x}}^{\mathrm{TM}}=+\frac{\sqrt{\epsilon_{m} 2}}{a(p)} \frac{m}{\sqrt{m^{2} \frac{b(p)}{a(p)}+n^{2} \frac{a(p)}{4 b(p)}}} \\
& N_{e_{y}}^{\mathrm{TM}}=+\frac{\sqrt{\epsilon_{m} 2}}{2 b(p)} \frac{n}{\sqrt{m^{2} \frac{b(p)}{a(p)}+n^{2} \frac{a(p)}{4 b(p)}}}, \\
& m=0,1,2, \cdots ; \\
& n=1,3, \cdots \text { (odd). } \\
& N_{e_{x}}^{\mathrm{TE}}=+\frac{2}{2 b(p)} \frac{n}{\sqrt{m^{2} \frac{b(p)}{a(p)}+n^{2} \frac{a(p)}{4 b(p)}}} \\
& N_{e_{y}}^{\mathrm{TE}}=+\frac{2}{a(p)} \frac{m}{\sqrt{m^{2} \frac{b(p)}{a(p)}+n^{2} \frac{a(p)}{4 b(p)}}}, \\
& m=1,2,3, \cdots ; \\
& n=1,3, \cdots \text { (odd). }
\end{aligned}
$$

\section{APPENDIX III \\ EXCITATION COEFFICIENTS}

Using the modal expansions derived for the ports in Appendix II, the coefficients $\gamma$, defined in (16) are needed to model the excitation. For a port placed at $x=0$ of Fig. 1, we use (37), and solve the integral in (16) to obtain

$$
\gamma_{m, n}^{(r)}=\left\{\begin{array}{l}
0, \quad n \neq 0 \\
N_{e_{x}}^{\xi} b(r), \quad n=0
\end{array}\right.
$$

where $b(r)$ is the width of the $r$ th line, as shown in Fig. 1, and the normalization coefficients $N_{e_{x}}^{\xi}$ are defined in (38). In the same way, for a port placed at $x=a$, we use (40) to obtain

$$
\gamma_{m, n}^{(r)}=\left\{\begin{array}{l}
0, \quad n \neq 0 \\
N_{e_{x}}^{\xi} \sin \left(\frac{m \pi}{2}\right) b(r), \quad n=0
\end{array}\right.
$$

where the normalization coefficients are those of (41). In addition, for $y$-directed ports, we can simply replace in the above relations the quantities associated to the $x$-axis with the corresponding quantities associated to the $y$-axis. For a port placed at $y=0$, we, therefore, write

$$
\gamma_{m, n}^{(r)}=\left\{\begin{array}{l}
0, \quad m \neq 0 \\
N_{e_{y}}^{\xi} a(r), \quad m=0
\end{array}\right.
$$

with the normalization coefficients in (43). Finally, for a port placed at $y=b$, one obtains

$$
\gamma_{m, n}^{(r)}=\left\{\begin{array}{l}
0, \quad m \neq 0 \\
N_{e_{y}}^{\xi} \sin \left(\frac{n \pi}{2}\right) a(r), \quad m=0
\end{array}\right.
$$

with the normalization coefficients given in (45).

\section{REFERENCES}

[1] T. G. Livernois and P. B. Katehi, "A generalized method for deriving the space-domain Green's function in a shielded, multilayer substrate structure with applications to MIS slow-wave transmission lines," IEEE Trans. Microwave Theory Tech., vol. 37, pp. 1761-1767, Nov. 1989.

[2] G. V. Eleftheriades, J. R. Mosig, and M. Guglielmi, "An efficient mixed potential integral equation technique (MPIE) for the analysis of shielded MMIC's," in 25th European Microwave Conf., Bologna, Italy, Sept. 4-7, 1995.

[3] R. Faraji-Dana and Y. L. Chow, "Accurate and efficient CAD tool for the design of optimum packaging for (M)MIC's," Proc. Inst. Elect. Eng., vol. 142, pp. 81-88, Apr. 1995.

[4] S. Singh and R. Singh, "On the use of $\rho$-algorithm in series acceleration,” IEEE Trans. Antennas Propagat., vol. 39, pp. 1514-1517, Oct. 1991.

[5] J. C. Rautio and R. F. Harrington, "An electromagnetic time-harmonic analysis of shielded microstrip circuits," IEEE Trans. Microwave Theory Tech., vol. MTT-35, pp. 726-730, Aug. 1987.

[6] C. J. Railton and S. A. Meade, "Fast rigorous analysis of shielded planar filters," IEEE Trans. Microwave Theory Tech., vol. 40, pp. 978-985, May 1992.

[7] G. V. Eleftheriades, J. R. Mosig, and M. Guglielmi, "A fast integral equation technique for shielded planar circuits defined on nonuniform meshes," IEEE Trans. Microwave Theory Tech., vol. 44, pp. 2293-2296, Dec. 1996.

[8] N. Marcuvitz, Waveguide Handbook (MIT Radiation Laboratory Series). Boston, MA: Boston Tech., 1964.

[9] K. A. Michalski and J. R. Mosig, "Multilayered media Green's functions in integral equation formulations," IEEE Trans. Antennas Propagat., vol. 45, pp. 508-519, Mar. 1997.

[10] S. G. Pan and I. Wolff, "Scalarization of dyadic spectral Green's functions and network formalism for three-dimensional full-wave analysis of planar lines and antennas," IEEE Trans. Microwave Theory Tech., vol. 42, pp. 2118-2127, Nov. 1994.

[11] G. V. Eleftheriades and J. R. Mosig, "On the network characterization of planar passive circuits using the method of moments," IEEE Trans. Microwave Theory Tech., vol. 44, pp. 438-445, Mar. 1996.

[12] G. Matthaei, L. Young, and E. M. T. Jones, Microwave Filters, Impedance-Matching Networks, and Coupling Structures. Norwood, MA: Artech House, 1980.

[13] M. Guglielmi and A. Alvarez Melcon, "Multimode network analysis of planar transmission lines," IEEE Trans. Microwave Theory Tech., vol. 43, pp. 2621-2626, Nov. 1995. 
[14] M. Guglielmi, "A simple CAD procedure for microwave filters and multiplexers," IEEE Trans. Microwave Theory Tech., vol. 42, pp. 1347-1352, July 1994.

[15] M. Guglielmi and A. Alvarez Melcon, "Novel design procedure for microwave filters," presented at the 23rd European Microwave Conf., Madrid, Spain, Sept. 6-10, 1993.

[16] D. Chambers and J. D. Rhodes, "Asymmetric synthesis of microwave filters," in Proc. 11th European Microwave Conf., Amsterdam, The Netherlands, Sept. 7-11, 1981, pp. 105-110.

[17] K. Iguchi, M. Tsuji, and H. Shigesawa, "Negative coupling between $\mathrm{TE}_{01}$ and $\mathrm{TE}_{02}$ modes for use in evanescent-mode bandpass filters and their field-theoretic CAD," in IEEE MTT-S Int. Microwave Symp., San Diego, CA, May 1994, pp. 727-730.

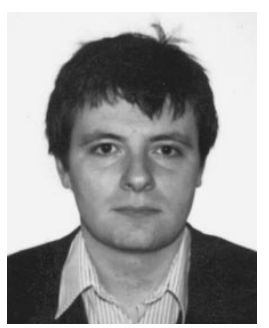

Alejandro Alvarez Melcón was born in Madrid, Spain, in 1965 . He received the Ingeniero Superior de Telecomunicaciones degree from the Polytechnic University of Madrid (UPM), Madrid, Spain, in 1991, and the Ph.D. degree in electrical engineering from the Swiss Federal Institute of Technology, Lausanne, Switzerland, in 1998

In 1988, he joined the Signal, Systems and Radiocommunications Department, UPM, as a research student, where he was involved in the design, testing, and measurement of broad-band spiral antennas for emergency medical system (EMS) equipment. From 1991 to 1993, he joined the Radio Frequency Systems Division, European Space Agency (ESA/ESTEC), Noordwijk, The Netherlands, where he was involved in the development of analytical and numerical tools for the study of waveguide discontinuities, planar transmission lines, and microwave filters. From 1993 to 1995, he joined the Space Division, Industry Alcatel Espacio, Madrid, Spain, and he worked at the ESA, where he collaborated in several ESA/ESTEC contracts. In 1995, he joined the Swiss Federal Institute of Technology, Ecole Polytechnique Fédérale de Lausanne, Lausanne, Switzerland, where he is currently working in the field of microstrip antennas and printed circuits for space applications. He holds an assistant position at the Electromagnetics and Acoustics Laboratory, Swiss Federal Institute of Technology, where he is currently collaborating in several ESA/ESTEC contracts and in the teaching activities of the laboratory.

Mr. Alvarez Melcón received the JINA Best Paper Award for the best contribution to the JINA'98 International Symposium on Antennas.

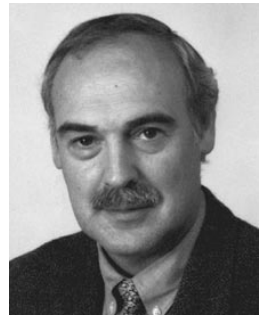

Juan R. Mosig (S'76-M'89-SM'94-F'99) was born in Cadiz, Spain. He received the electrical engineer degree from Universidad Politecnica de Madrid, Madrid, Spain, in 1973, and the Ph.D degree from Ecole Polytechnique Fédérale de Lausanne, Lausanne, Switzerland, in 1983.

In 1976, he joined the Electromagnetics and Acoustics Laboratory, Swiss Federal Institute of Technology, Ecole Polytechnique Fédérale de Lausanne. Since 1991, he has been a Professor at Ecole Polytechnique Fédérale de Lausanne. In 1984, he was a Visiting Research Associate at Rochester Institute of Technology, Rochester, NY. He has also held scientific appointments at universities of Rennes, France, Nice, France, Technical University of Denmark, and the University of Colorado at Boulder. He has authored four chapters in books on microstrip antennas and circuits. His research interests include electromagnetic theory, numerical methods, and microstrip antennas. He is co-organizer and lecturer of yearly short intensive courses in numerical electromagnetics in both Europe and the U.S.

Dr. Mosig is a member of the Swiss Federal Commission for Space Applications and is responsible for several research projects for the European Space Agency.

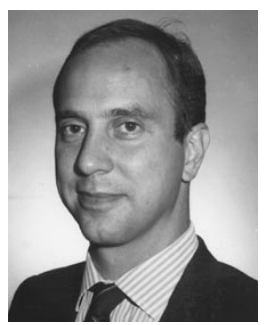

Marco Guglielmi was born in Rome, Italy, on December 17, 1954. He received the Laurea in Ingegneria Elettronica degree from the University of Rome "La Sapienza," Rome, Italy, in 1979, attended the Scuola di Specializzazione in Electtromagnetismo Applicato in 1980, received the M.S degree in electrical engineering from the University of Bridgeport, Bridgeport, CT, in 1982, and the $\mathrm{Ph} . D$. degree in electrophysics from Polytechnic University, Brooklyn, NY, in 1986.

From 1984 to 1986, he was an Academic Associate at Polytechnic University. From 1986 to 1988, he was an Assistant Professor at the New Jersey Institute of Technology, Newark, NJ. In 1988, he joined the RF System Division, European Space Research and Technology Center, Noordwijk, The Netherlands, where he is currently involved in the development of passive microwave components for space applications. $\mathrm{He}$ is currently with the European Space Research and Technology Center, Noordwijk, The Netherlands. His professional interests include the areas of solid-state devices and circuits, periodic structures, phased arrays, and millimeter-wave leaky-wave antennas, network representations of waveguide discontinuities, and microwave filtering structures.

Dr. Guglielmi was the recipient of a 1981 Fulbright Scholarship in Rome, Italy, and an Halsey International Scholarship Program (HISP) Scholarship from the University of Bridgeport. 